\title{
lodine-125 prostate seed brachytherapy in renal transplant recipients: an analysis of oncological outcomes and toxicity profile
}

\author{
Nadine Beydoun, BSc (Med), MBBS, FRANZCR', Joseph Bucci, BSc (Med), MBBS, FRACP, FRANZCR', David Malouf, BSc \\ (Med), MBBS, FRACS (Urol) $)^{2}$ \\ ISt George Hospital Cancer Care Centre, ${ }^{2}$ Department of Urology, St George Hospital, Kogarah, Australia
}

\begin{abstract}
Purpose: Prostate cancer is among the most common non-cutaneous neoplasms affecting renal transplant recipients (RTRs). Available treatments including radical prostatectomy and external beam radiotherapy carry a risk of damage to the transplanted kidney, ureters, or bladder. We assessed the safety and efficacy of Iodine- 125 ( $\left.{ }^{125} \mathrm{I}\right)$ prostate seed brachytherapy as an alternative to surgery and radiotherapy in these individuals.

Material and methods: We retrospectively reviewed our brachytherapy database to identify patients with a prior history of renal transplantation, who had undergone seed implantation for localized prostate cancer. Long term PSA control and treatment related toxicity, including graft dysfunction, urinary, rectal, and sexual complications, were assessed and compared with published outcomes for surgery and external beam radiotherapy.

Results: Of 1054 patients treated with permanent seed implantation from 2002-2012, we identified four who had a prior history of renal transplantation. Mean time from renal transplantation to prostate cancer diagnosis was 13 years. Mean follow-up after seed implantation was 44 months (range 12-60 months). All four patients remain free of PSA progression. No peri-operative complications were experienced following seed implantation, and all four patients continued to have normal graft function. Long term urinary and rectal function scores were comparable to reported outcomes for seed brachytherapy in the non-transplant population.

Conclusions: ${ }^{125}$ I prostate seed brachytherapy is associated with high rates of biochemical control and minimal toxicity to the renal graft in RTRs. This treatment should be considered as an alternative to surgery in managing RTRs with localized prostate cancer.
\end{abstract}

Key words: brachytherapy, ${ }^{125} \mathrm{I}$, prostate cancer, renal transplant, seeds.

\section{Purpose}

Prostate cancer is the commonest malignancy, and second biggest contributor to cancer mortality among men. Interstitial prostate brachytherapy, surgery, and external beam radiotherapy are widely accepted curative treatment options for organ confined prostate cancer [1]. Renal transplant recipients (RTRs) are known to have a much higher risk of developing malignancies, due to chronic immunosuppression and antigenic stimulation [2]. Whilst skin cancers and lymphoproliferative disorders constitute the majority of these, genitourinary (GU) malignancies are the next most common neoplasms affecting RTRs [2,3]. Some studies have reported a 2 to 5 fold higher incidence of prostate cancer in RTRs than the general population [3-6]. Also, an increasing number of transplant recipients are being diagnosed with prostate cancer, attributable in part to the growing acceptance of PSA screening, but also due to longer survival of transplant recipients, and an increase in the number of older male transplant patients [7]. Prostate cancer also tends to occur at a younger age in transplanted patients than the general population [2].

These trends suggest that prostate cancer may become an important contributor to morbidity and mortality in this group, who are now better managed and less likely to die of post-transplant infections or cardiovascular complications. The available evidence describing prostate cancer treatment outcomes in renal transplant recipients however, remains very limited. Published work has mainly consisted of case reports and small surgical series, with heterogeneity in the outcomes reported and in general, less focus on long term cancer control as much as the immediate peri-operative course. 
Radical prostatectomy has been supported as a treatment for localized prostate cancer in renal transplant patients, but still carries a risk of injury to the transplanted kidney, ureters or bladder. Damage to the graft can result from blood loss during the operative procedure or direct trauma due to the position of the transplanted kidney in the iliac fossa. Being immunosuppressed, these patients are also at a higher risk of infection and problems with wound healing [7]. Various approaches to prostatectomy have been described in RTRs, including radical retropubic prostatectomy (RRP), laparoscopic, and transperineal techniques, but limited follow-up and highly selected patient cohorts (mostly Gleason 6, clinical T1c, and mean PSA levels less than $5 \mathrm{ng} / \mathrm{mL}$ ) in these series raise uncertainty as to long term biochemical outcomes and toxicity with a surgical strategy [8].

Wagener et al. described a six-port transperitoneal approach to robot-assisted laparoscopic radical prostatectomy (RALP) in a 71 year old renal transplant patient with Gleason 7 localized prostate cancer [9]. In this patient, pelvic lymph node dissection was only undertaken on the contralateral side to the transplanted kidney. The procedure took greater than 3.5 hours to perform with a blood loss of $300 \mathrm{~mL}$. Though the post-operative course was uncomplicated, no data on long term PSA control or toxicity was provided. Smith et al. also retrospectively identified all patients who had undergone robot-assisted radical prostatectomy (RARP) for localized prostate cancer $(n=228)$, who were transplant recipients $(n=3)$. Two patients required modification of their port placement in order to avoid damaging the renal allograft. All three patients were reported to have undetectable PSAs and be continent at twelve months follow-up [10].

External beam radiotherapy to the prostate has also been used in RTRs with disappointing results, including biochemical relapse free survival rates of only $50 \%$, and graft failure or distal ureteric obstruction in 38\% [2,11]. The renal allograft location in the pelvis makes it technically challenging to deliver high doses of radiotherapy whilst avoiding damage to the graft. Contemporary definitive external beam radiotherapy regimens generally aim to deliver doses exceeding $70 \mathrm{~Gy}$. The kidney is a highly radiosensitive organ, with increasing risk of clinically significant renal dysfunction being induced beyond mean doses of $15 \mathrm{~Gy}$ [12]. Dose to the femoral heads is also of particular importance in RTRs, as the use of corticosteroids and other immunosuppressive drugs in this group places them at a higher risk of avascular necrosis than the general population [2]. Attempts to minimize dose to organs at risk have included dose reduction or compromising the planning target volume (PTV), both of which could diminish the efficacy of treatment [2]. While surgery is often favored for these reasons, the risks of surgery mentioned earlier, together with the possibility that adjuvant radiotherapy may not be altogether avoided in those with adverse pathological features following prostatectomy, are factors which warrant serious consideration [2]. Moreover, the potential for future transplants in the event of graft failure is something that must be considered in managing RTRs with prostate cancer either with a surgical or radiotherapeutic approach.
A third option in RTRs, which has been used to treat localized prostate cancer for decades, and has the appeal of minimal invasiveness and a favorable toxicity profile, is transperineal interstitial permanent prostate brachytherapy $[1,13,14]$. A key advantage of prostate brachytherapy is that it enables a highly conformal dose distribution, with rapid dose fall off with increasing distance from the radiation sources, thereby allowing preservation of both oncological efficacy and functioning of the renal graft. Published results on the utility of seed brachytherapy in RTRs are lacking. We report on our experience and long term outcomes in RTRs treated with Iodine-125 $\left({ }^{125} \mathrm{I}\right)$ prostate seed brachytherapy at an established institution.

\section{Material and methods}

\section{Patient characteristics}

Four renal transplant recipients were identified as having undergone seed implantation at the St George Hospital Cancer Care Centre between 2002-2012. Reasons for kidney transplantation included chronic glomerulonephritis $(n=2)$, hypertensive nephropathy $(n=1)$, and polycystic kidney disease $(n=1)$. All patients were receiving maintenance immunotherapy. Two patients had a previous diagnosis of immunosuppression-related non-melanomatous skin cancer, including one with metastatic cutaneous squamous cell carcinoma to the parotid and upper cervical lymph nodes treated with surgery and radiotherapy. All patients had functioning grafts at the time of prostate cancer diagnosis, with a mean serum creatinine clearance level of $113 \mu \mathrm{mol} / \mathrm{L}$ (range 80-145). One patient had been experiencing chronic graft rejection since undergoing kidney transplantation two years prior to seed brachytherapy, but his creatinine level had stabilized at $145 \mu \mathrm{mol} / \mathrm{L}$ before undergoing seed implantation. None of the patients received neo-adjuvant or adjuvant hormonal therapy. Table 1 illustrates demographic and tumor characteristics of the treated cohort.

\section{Database}

The department's prospective brachytherapy database BrachyNet was reviewed to identify all renal transplant recipients who were treated with ${ }^{125}$ I prostate seed brachytherapy from the commencement of our program in October 2002, until June 2012. Implantation technique is described. Data on PSA control and treatment toxicity was obtained from BrachyNet. The main endpoints of interest were PSA progression free survival and long term treatment related toxicity, including urinary and rectal function, potency, and graft function. The results of validated questionnaires designed to capture information on patients sexual, urinary, and rectal function at baseline and last available follow-up for each patient were compared. Questionnaires comprised the International Index of Erectile Function (IIEF), International Prostate Symptom Score (IPSS), and Expanded Prostate Cancer Index Composite (EPIC) Bowel Assessment [15-17]. Information on graft function, including creatinine clearance $(\mu \mathrm{mol} / \mathrm{L})$ before and after seed implantation was obtain- 
ed from patients clinical records and correspondence from their renal physician. PSA progression free survival was calculated from the date of seed implantation to the date of PSA progression, or last available follow-up. The Phoenix definition (nadir PSA + 2.0) was used to define PSA progression [18]. Descriptive statistics (mean, median, range, standard deviation [SD]) were used to summarize patient, tumor, and treatment details.

\section{Seed implantation technique}

All implants were undertaken according to a preplanned, modified peripherally loaded seed pattern, as described previously by the Seattle group [19]. Under general anesthesia, patients were placed in the dorsal lithotomy position, and the bladder filled with $250 \mathrm{~mL}$ of dilute contrast via an indwelling catheter. Transperineal needle placement and deployment of pre-loaded ${ }^{125}$ I seeds was undertaken using transrectal ultrasound and fluoroscopic guidance. Median operative time for the cohort was 70 minutes (range 55-90 minutes). The indwelling catheter was removed during recovery and all patients were discharged on the day of implantation. All four patients experienced an unremarkable post-operative course. Figure 1 is a post-operative computed tomography scan which illustrates the distribution of radioactive ${ }^{125}$ I seeds in relation to the allograft in one study patient.

Minimum follow-up for the cohort was 12 months (range 12-60 months).

\section{Results}

\section{Toxicity}

Mean International Prostate Symptom Score (IPSS) at last follow-up was $5 \pm 4.1$, compared with $3 \pm 3.6$ at baseline. Mean International Index of Erectile Function (IIEF) score was $8 \pm 11.7$, compared with $5 \pm 4.6$ at baseline, and mean Expanded Prostate Cancer Index Composite (EPIC) bowel assessment score was $22 \pm 4.7$ compared with $23 \pm 3.8$ at baseline [15-17]. No patient experienced graft dysfunction following seed implantation. Mean creatinine clearance level post-operatively was 98 umol/L (range 76-120). Figure 2 illustrates the difference in IPSS, IIEF, and EPIC scores from baseline to last available follow-up for each of the four patients. There was no significant deviation in bowel function from baseline to post-treatment. All four patients had erectile dysfunction predating their brachytherapy. Three patients experienced a return of urinary function to near baseline levels. The fourth patient was continuing to experience clinically significant voiding symptoms compared to baseline, but was only 12 months out from seed implantation. His symptoms were managed successfully with alpha blockers. No patient required urinary catheterization at any point following seed implantation.

\section{PSA control}

After a mean follow-up of 44 months (range 12-60 months), all patients were alive with no evidence of PSA relapse or distant failure. One patient was being treated
Table 1. Patient and tumor characteristics

\begin{tabular}{|c|c|}
\hline Factor & $N(\%)$ \\
\hline \multicolumn{2}{|l|}{ Age } \\
\hline Median (range) & $64(61-66)$ \\
\hline \multicolumn{2}{|l|}{ Smoking status } \\
\hline Current & $0(0)$ \\
\hline Never & $3(75)$ \\
\hline Ex-smoker & $1(25)$ \\
\hline \multicolumn{2}{|l|}{ Comorbidities } \\
\hline Hypertension & $3(75)$ \\
\hline Ischemic heart disease & $2(50)$ \\
\hline Diabetes mellitus & $1(25)$ \\
\hline Other cancer & $2(50)$ \\
\hline \multicolumn{2}{|l|}{ Years from transplant } \\
\hline Median (range) & $13(6-17)$ \\
\hline \multicolumn{2}{|c|}{ Immunosuppressant therapy } \\
\hline Cyclosporine & 2 \\
\hline Tacrolimus & 1 \\
\hline Sirolimus & 1 \\
\hline Mycophenolate mofetil & 1 \\
\hline Prednisone & 3 \\
\hline \multicolumn{2}{|l|}{ Clinical Stage } \\
\hline $\mathrm{T1C}$ & $3(75)$ \\
\hline T2a & $1(25)$ \\
\hline \multicolumn{2}{|l|}{ iPSA } \\
\hline Mean \pm SD & $8.9 \pm 3.8$ \\
\hline \multicolumn{2}{|l|}{ Gleason score } \\
\hline 7 & 3 \\
\hline 8 & 1 \\
\hline \multicolumn{2}{|l|}{ Hormonal therapy } \\
\hline Yes & $0(0)$ \\
\hline No & $4(100)$ \\
\hline \multicolumn{2}{|l|}{ Baseline IPSS (0-35) } \\
\hline Mean \pm SD & $3 \pm 3.6$ \\
\hline \multicolumn{2}{|l|}{ Baseline IIEF Score (0-25) } \\
\hline Mean \pm SD & $5 \pm 4.6$ \\
\hline \multicolumn{2}{|c|}{ Baseline EPIC Bowel Score (8-62) } \\
\hline Mean \pm SD & $23 \pm 3.8$ \\
\hline
\end{tabular}

$N$ - number of patients, iPSA - initial prostate specific antigen level, SD - standard deviation, IPSS - International Prostate Symptoms Score, IIEF - International Index of Erectile Function, EPIC - Expanded Prostate Cancer Index

with surgery and radiotherapy for metastatic cutaneous squamous cell carcinoma to the axilla more than three years after undergoing prostate brachytherapy and his PSA has remained undetectable. Of patients who had sufficient post-treatment follow-up to achieve a PSA nadir $(n=3)$, median nadir PSA was 0 (range $0-0.14 \mathrm{ng} / \mathrm{mL}$ ).

\section{Discussion}

Genitourinary (GU) malignancies are becoming an increasingly important source of morbidity and mortality 

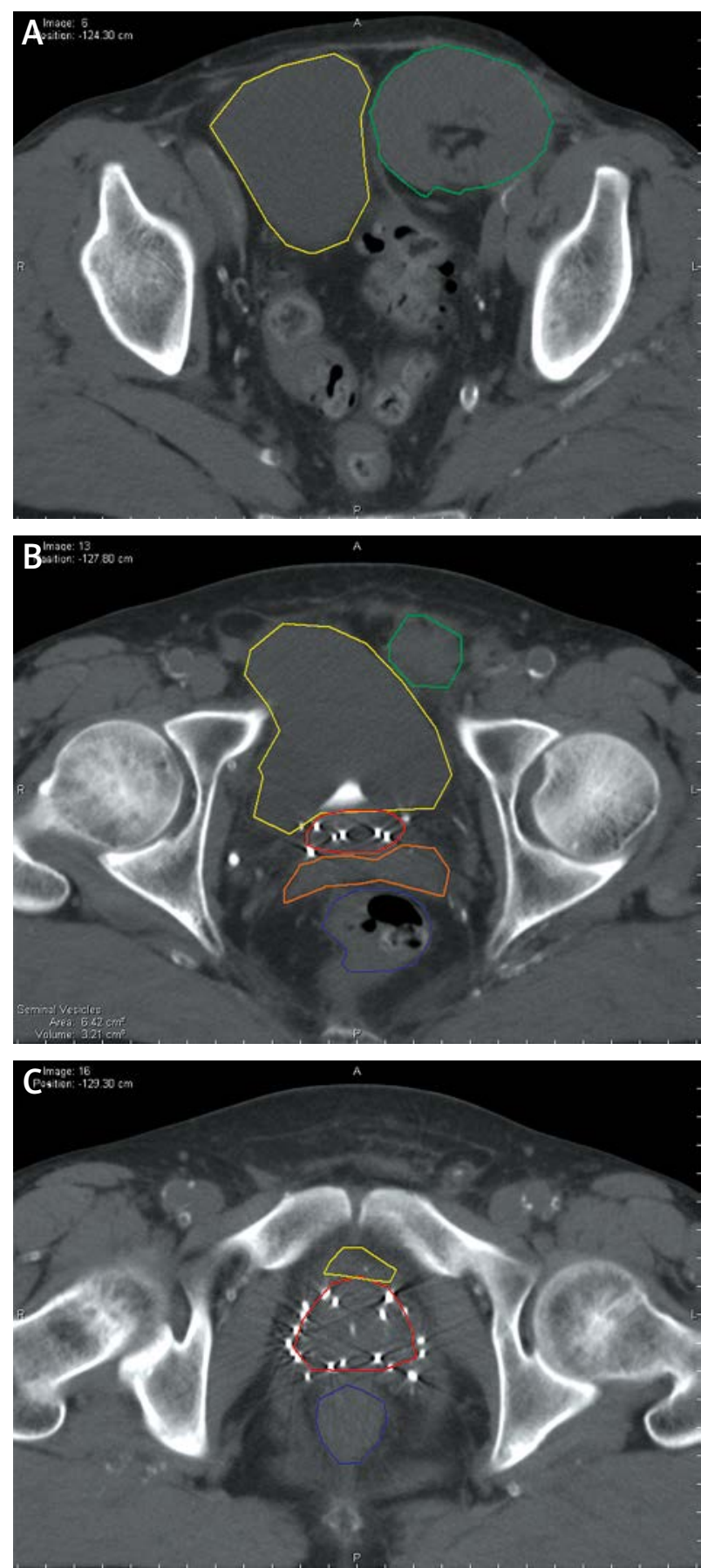

Fig. 1. Distribution of radioactive Iodine-125 seeds on computed tomography scan in relation to the allograft in one study patient. (A) Mid-portion of renal graft, (B) inferior portion of graft and superior-most intraprostatic seeds, (C) mid-portion of prostate.

Green - renal allograft; red - prostate; yellow - bladder; orange - seminal vesicles; blue - rectum

in RTRs [7]. Because of advances in immunosuppressive therapy for RTR, half-lives for both living and cadaveric grafts have almost doubled, to 21.6 years, and 13.8 years, respectively. Management of GU tumors can be a challenge in these patients, as available definitive therapies can significantly impact on graft survival and function.
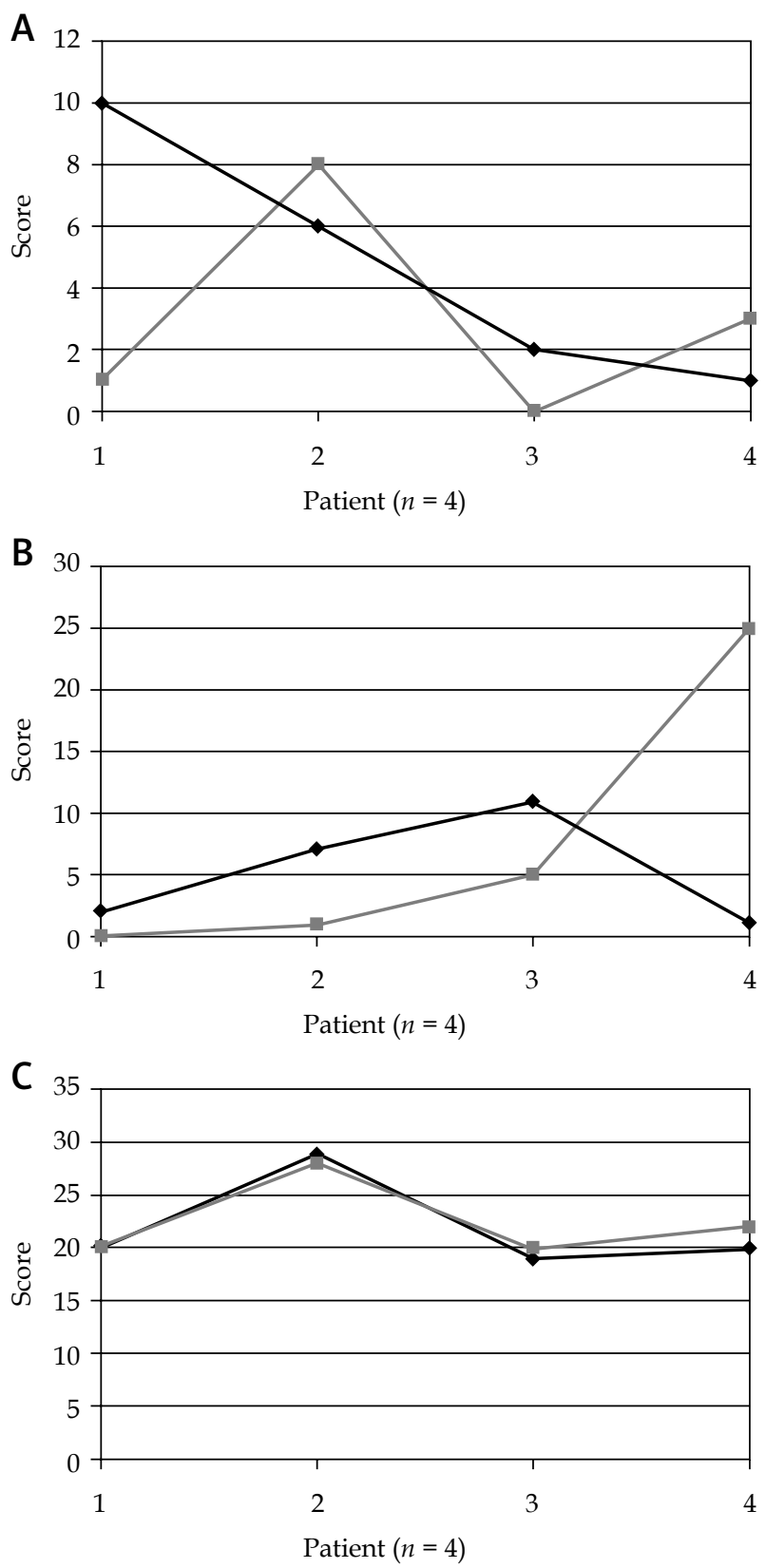

Fig. 2. (A) Difference in International Prostate Symptom Score (top), (B) International Index of Erectile Function (middle), and (C) Expanded Prostate Cancer Index Composite bowel assessment scores (bottom) from baseline to last available follow-up for each of the four patients.

Grey line - baseline score; black line - follow-up score

The likelihood of death with a functioning graft is three times greater in RTRs with a GU malignancy than in those without [20].

Problems can be encountered when attempting to treat RTRs with external beam radiotherapy, due to proximity of the graft to the treatment region, and the inherent radiosensitivity of this organ to even modest doses of radiation [12]. Whilst successful treatment of the prostate alone with definitive radiotherapy to $70 \mathrm{~Gy}$ has been reported in RTRs, this was associated with biochemical recurrence in $25 \%$, as well as graft failure in one patient 
and ureteric obstruction in a further two, which could precipitate future graft dysfunction [11].

Though surgery has traditionally been considered the preferred treatment approach, graft function can also be threatened through prolonged operative times and blood loss, intra-operative trauma to the pelvic renal graft, or post-operative bladder dysfunction [7]. Moreover, while pelvic lymph node dissection may not be routinely performed during RRP, there is some evidence to suggest that patients on more heavy maintenance immunosuppressive therapy are more likely to present with locally advanced tumors and lymph node invasion [6]. This has two important implications. Firstly, oncological outcomes could be compromised with surgery in those with nodal involvement as comprehensive pelvic nodal dissection may be limited by the location of the graft. Secondly, those with unfavorable histopathology such as margin involvement or seminal vesicle invasion may not be able to avoid post-operative radiotherapy, which would compound the toxicity of therapy, including risk of damage to the renal graft. Both these modalities also require consideration of the possibility of future graft failure and the potential for new graft placement. Approximately one third of RTRs will require a new transplant due to graft failure, and this can be complicated by previous radiation or surgery $[21,22]$.

Prostate brachytherapy can potentially overcome the complications experienced with external beam radiotherapy by enabling highly conformal radiation treatment to be delivered to the target volume, with a steep dose gradient surrounding this region, thereby limiting toxicity to neighboring organs at risk. Although seed brachytherapy is considered a standard treatment option for localized prostate cancer, outcomes have not been specified for RTRs. A recent review by Coombs et al. described prostate cancer outcomes in seven of their brachytherapy patients who were also organ transplant recipients [23]. Three of these were renal transplant patients, with the remainder being heart transplants. While the difficulties mentioned above in treating renal transplant recipients with respect to the graft being in close proximity to the radiation field are less of an issue with heart transplant patients, of relevance is that after a minimum follow-up of three years, the transplanted patient subset had comparable rates of biochemical control $85 \%$ ) to non-transplant patients $(n=307)$ treated at the same center [23]. Biochemical control rates were equally high in our series, with all four patients being free of prostate cancer relapse at a mean follow-up of 44 months. Moreover, RTRs who underwent seed implantation at our institution continued to have normal graft function following treatment according to pre and post-implant serum creatinine clearance levels.

Temporary urinary sequel in the form of an increased IPSS have been reported to occur in approximately $20 \%$ of patients beyond 12 months after permanent seed brachytherapy [24]. These have generally comprised of flares, and the majority of these patients will respond to alpha blockers or anticholinergics. Chronic IPSS increases are seen in less than $5 \%$ of brachytherapy patients [24]. Of the patients beyond 12 months of implantation in our study, all reported a follow-up IPSS that was within two points of their baseline score. All four patients had no significant deviation in their EPIC scores from baseline. The impact of treatment on sexual function was difficult to assess in this cohort, due to significant erectile dysfunction existing prior to seed implantation. As such, it is likely that long term erectile function after seed implantation would be confounded by other medical conditions in this population.

Our mean operative time of 75 minutes in the seed cohort also compares favorably to surgical series, where operative times more than twice this duration have been reported [3,9]. Due to its minimal invasiveness compared to prostatectomy, all patients were able to be discharged on the same day as their seed implant and experienced no complications during their post-operative course.

Finally, though immunosuppression has been implicated in malignant cell growth, it remains uncertain as to whether prostate cancer runs a more aggressive course in RTRs [25]. Our patients all presented with localized tumors and were free of disease relapse at last available follow-up. Nevertheless, a larger patient cohort and longer follow-up would be required to ascertain the natural history of this disease in RTRs.

\section{Conclusions}

${ }^{125}$ I prostate seed brachytherapy was associated with favourable biochemical outcomes and minimal toxicity, and should be considered as an alternative to surgery in renal transplant patients.

\section{Disclosure}

Authors report no conflict of interest.

\section{References}

1. Skowronek J. Low-dose-rate or high-dose-rate brachytherapy in treatment of prostate cancer-between options. I Contemp Brachytherapy 2013; 5: 34-41.

2. Detti B, Scoccianti S, Franceschini D et al. Adjuvant radiotherapy for a prostate cancer after renal transplantation and review of the literature. Jpn J Clin Oncol 2011; 41: 1282-1286.

3. Maestro MA, Gomez AT, Gregorio SA et al. Laparoscopic transperitoneal radical prostatectomy in renal transplant recipients: a review of the literature. BJU Int 2009; 105: 844-848.

4. Cormier L, Lechevallier E, Barrou B et al. Diagnosis and treatment of prostate cancers in renal-transplant recipients. Transplantation 2003; 75: 237-239.

5. Birkeland SA, Storm HH, Lamm LU et al. Cancer risk after renal transplantation in the Nordic countries, 1964-1986. Int J Cancer 1995; 60: 183-189.

6. Kleinclauss F, Gigante M, Neuzillet Y et al. Prostate cancer in renal transplant recipients. Renal Transplantation Committee of the French Urological Association (AFU). Nephrol Dial Transplant 2008; 23: 2374-2380.

7. Hoda MR, Hamza A, Greco F et al. Management of localized prostate cancer by retropubic radical prostatectomy in patients after renal transplantation. Nephrol Dial Transplant 2010; 25: 3416-3420.

8. Elkentaoui H, Robert G, Pasticier G et al. Therapeutic management of de novo urological malignancy in renal transplant recipients: the experience of the French Department of Urology and Kidney Transplantation from Bordeaux. Urology 2010; 75: 126-132. 
9. Wagener N, Nyarangi-Dix JN, Teber D et al. Applicability of robot-assisted laparoscopic radical prostatectomy in renal allograft recipients. Transplant Proc 2012; 44: 1287-1292.

10. Smith DL, Jellison FC, Heldt JP et al. Robot-assisted radical prostatectomy in patients with previous renal transplantation. J Endourol 2011; 25: 1643-1647.

11. Mouzin M, Bachaud JM, Kamar N et al. Three-dimensional conformal radiotherapy for localized prostate cancer in kidney transplant recipients. Transplantation 2004; 78: 1496-1500.

12. Marks LB, Yorke ED, Jackson A et al. Use of normal tissue complication probability models in the clinic. Int J Radiat Oncol Biol Phys 2010; 76: S10-S19.

13. Aronowitz JN, Rivard MJ. The phylogeny of permanent prostate brachytherapy. J Contemp Brachytherapy 2013; 5: 89-92.

14. Merrick GS, Butler WM, Grimm P et al. Permanent prostate brachytherapy extracapsular radiation dose distributions: analysis of a multi-institutional database. J Contemp Brachytherapy 2013; 5: 117-121.

15. Rosen RC, Riley A, Wagner G et al. The international index of erectile function (IIEF): a multidimensional scale for assessment of erectile dysfunction. Urology 1997; 49: 822-830.

16. Wei JT, Dunn RL, Litwin MS et al. Development and validation of the expanded prostate cancer index composite (EPIC) for comprehensive assessment of health-related quality of life in men with prostate cancer. Urology 2000; 56: 899-905.

17. Barry MJ, Fowler FJ, O'Leary MP et al. The American Urological Association symptom index for benign prostatic hyperplasia. J Urol 1992; 148: 1549-1557.

18. Roach M, Hanks G, Thames H Jr et al. Defining biochemical failure following radiotherapy with or without hormonal therapy in men with clinically localized prostate cancer: recommendations of the RTOG-ASTRO Phoenix Consensus Conference. Int J Radiat Oncol Biol Phys 2006; 65: 965-974.

19. Blasko JC, Mate T, Sylvester JE et al. Brachytherapy for carcinoma of the prostate: Techniques, patient selection, and clinical outcomes. Semin Radiat Oncol 2002; 12: 81-94.

20. Muruve NA, Shoskes DA. Genitourinary malignancies in solid organ transplant recipients. Transplantation 2005; 80: 709716.

21. Hariharan S, Johnson CP, Bresnahan BA et al. Improved graft survival after renal transplantation in the United States, 1998 to 1996. N Engl J Med 2000; 342: 605-612.

22. Hafron J, Fogarty JD, Wiesen A et al. Surgery for localized prostate cancer after renal transplantation. BJU Int 2005; 95: 319-322.

23. Coombs CC, Hertzfeld K, Barrett W. Outcomes in transplant patients undergoing brachytherapy for prostate cancer. $\mathrm{Am}$ J Clin Oncol 2012; 35: 40-44.

24. Crook J, Fleshner N, Roberts C et al. Long term urinary sequelae following 125-Iodine prostate brachytherapy. J Urol 2008; 179: 141-146.

25. Bustami RT, Ojo AO, Wolfe RA et al. Immunosuppression and the risk of post-transplant malignancy among cadaveric first kidney transplant recipients. Am J Transplant 2004; 4: 87-93. 